halt muss nicht bei einem der Kooperationspartner des GlobalMathNetwork stattfinden, aber wenn es sich um einen Kooperationspartner handelt, dann ist die Unterstützung durch den Gastgeber maximal gewährleistet. Insbesondere fallen dann keine Studiengebühren an.

Die dritte Neuerung, auf die wir sehr viel Wert legen, ist die Stärkung der Zusammenarbeit mit den Nachbarwissenschaften. Wir haben dafür ein neues Programmformat konzipiert, die Interdisciplinary Research Units, kurz IRUs, die an ausgewählten Schnittstellen zu den Nachbarwissenschaften angesiedelt sein werden. Eingebettet in ein Netzwerk von etablierten Wissenschaftlerinnen und Wissenschaftlern von beiden Seiten der beteiligten Fächer wird jeweils eine Nachwuchsgruppe aufgebaut, typischerweise ausgestattet mit einer befristeten W2-Professur und je einer Postdoc- und einer Promotionsstelle. Diese IRUs sollen paritätisch von beiden Kooperationspartnern finanziert werden. Entsprechende Kooperationen wurden vereinbart mit den Materialwissenschaften, mit der Mathematischen Physik und - in Form von sogar drei solcher Gruppen - den Lebenswissenschaften. Im Bereich der Lebenswissenschaften sind die dazugehörigen Berufungsverfahren gemeinsam mit ImmunoSensation, einem weiteren Bonner Exzellenzcluster, schon auf den Weg gebracht und es liegt bereits die erste Rufannahme vor. Professor Hasenauer wird sich mit seiner Arbeitsgruppe „Biomathematik“ auf die Entwicklung von Methoden zu datengetriebenen Modellierungen biologischer Prozesse konzentrieren. Damit kann man biologische Hypothesen überprüfen und zukünftige Experimente maßgeschneidert auswählen.

\section{Was wünschen Sie sich für die Zukunft des Hausdorff} Center?

Zunächst ist mein Wunsch natürlich, dass die Arbeitsbedingungen hier in Bonn nach wie vor gute Mathematik ermöglichen und dass wir die besten Kräfte der Welt ob nun als Gäste oder dauerhaft - zu uns bringen. Weiterhin wünsche ich mir, dass wir bei großen wichtigen Problemen der Mathematik, aber auch bei mathematischen Problemen der Gesellschaft, Wesentliches beitragen in den nächsten Jahren, dass wir ein dynamisches Projekt bleiben, uns also weiterentwickeln und immer wieder neue Schwerpunkte setzen. Wir können nicht die komplette Mathematik abdecken, aber wir sollten unsere Energie so bündeln, dass wir bei allen wichtigen Fragen zur Forschung entscheidend mitwirken.

\author{
Stefan Hartmann \\ Wissenschaftlicher Mitarbeiter Hausdorff Center for Mathematics (HCM), \\ Rheinische-Friedrich-Wilhelms-Universität Bonn \\ Endenicher Allee 6253115 Bonn \\ stefan.hartmann@hcm.uni-bonn.de
}

\title{
Was ist eigentlich die Hausdorff School?
}

\author{
Stefan Hartmann
}

Die Zeit zwischen Promotion und Professur ist eine bislang oft vernachlässigte Übergangsphase. Gerade dort werden die entscheidenden Weichen für die berufliche und private Zukunft gestellt. Die jungen Wissenschaftlerinnen und Wissenschaftler entwickeln nicht nur ihre ersten eigenen Forschungsprogramme einschließlich Personalverantwortung, sondern müssen auch ihre ersten Vorlesungen halten und Seminare leiten - dabei oft ohne jegliche Vorbereitung, geschweige denn systematisches Training. Ohne eine Mentorin oder einen Mentor und ohne die Ausbildung von allgemeinen Lehrkompetenzen gleichen diese Anforderungen für viele einem „Sprung ins kalte Wasser". Die Bonner Hausdorff School for Advanced
Studies in Mathematics möchte diesen Missstand beheben und eine Brücke schlagen zwischen Promotion und Professur, aber auch zu einer Karriere außerhalb der Universität.

Das Angebot der Hausdorff School richtet sich an Postdoktorandinnen und Postdoktoranden weltweit und setzt sich aus mehreren Komponenten zusammen: einem wissenschaftlichen Kursprogramm in Form von Sommeroder Winterschulen, einem Fortbildungsangebot zur Erlangung beruflicher Kernkompetenzen sowie einem Trainingskonzept für die akademische Lehre inklusive eines Mentorenprogramms. Das Kursprogramm besteht aus bis zu zehn einwöchigen Schulen pro Jahr. Das Themenspektrum ist dabei weit gefächert und spiegelt die Mathematik 
in ihrer gesamten Breite inklusive ihrer Anwendungen wider. Der Fokus wird stets auf aktuelle wissenschaftliche Entwicklungen gelegt. Für jede Schule werden zwei bis vier führende Vertreterinnen und Vertreter des jeweiligen Gebietes als Dozentinnen und Dozenten angeworben, die zum Teil aus Bonn, zum Großteil aber von anderen Universitäten kommen. Alle Veranstaltungen sind offen für Postdocs aus aller Welt, und das Hausdorff Center übernimmt die Aufenthalts- und Reisekosten für bis zu 25 externe Teilnehmerinnen und Teilnehmer. Die Schulen dienen dabei nicht nur der fachlichen Ausbildung, sondern auch der Netzwerkbildung und dem Austausch mit gleichgesinnten jungen Mathematikerinnen und Mathematikern.

Neben dem fachlichen Kursprogramm bietet die Hausdorff School umfangreiche Fortbildungen zur Erlangung beruflicher Kernkompetenzen an, wie etwa Medien-, Vortrags-, Interview- und Bewerbungstrainings sowie Informationsveranstaltungen, beispielsweise zur Drittmittelförderung. Bei diesen Veranstaltungen geben Expertinnen und Experten zum Beispiel Hinweise zu einem optimalen Bewerbungsportfolio oder Insidertipps aus Kommissionsarbeiten.

Mit seinem Postdoc-Mentorenprogramm hat die Hausdorff School zudem ein ambitioniertes Ausbildungskonzept für die akademische Lehre entwickelt. Das Ziel ist klar: Auch das Unterrichten im Hörsaal kann (und sollte) man lernen! In diesem Programm planen ein Postdoc und eine Professorin oder ein Professor gemeinsam eine Vorlesung für ein Semester. Der Postdoc hält mindestens die Hälfte der Vorlesungen und die Professorin oder der Professor gibt Feedback und Ratschläge. Dieser Prozess wird von einer Kommission beratend begleitet. Nach einer erfolgreichen Teilnahme werden den Nachwuchswissenschaftlerinnen und -wissenschaftlern Zertifikate ausgestellt.

Es gibt in Deutschland dringenden Bedarf zur Gewinnung und Fortbildung des mathematischen Nachwuchses aus der ganzen Welt. Viele Postdocs gehen von Deutschland aus in die USA; nur wenige kommen bislang aus anderen Ländern gezielt nach Deutschland. Ein solch umfangreiches Programm kann natürlich nicht an jedem

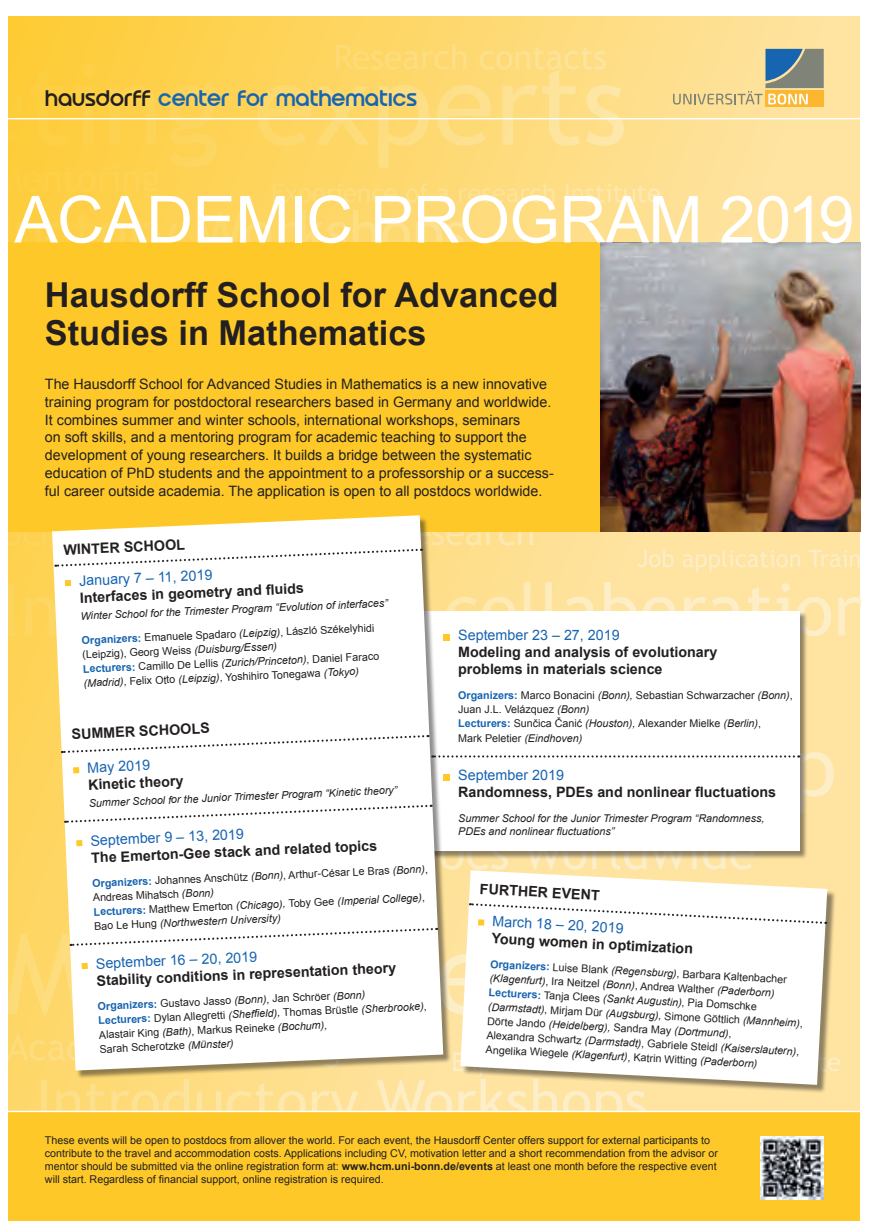

mathematischen Fachbereich in Deutschland angeboten werden. Daher hat sich das Hausdorff Center als mathematischer Exzellenzcluster zum Ziel gesetzt, der mathematischen Community insgesamt mit der Hausdorff School einen zentralen Service anzubieten, den alle Postdocs weltweit nutzen können und von dem ganz Deutschland als mathematischer Standort profitiert. Auf diese Weise möchte die Hausdorff School weltweit eine Vorreiterrolle spielen und durch seine Programme die mathematische Forschung und Lehre nachhaltig verbessern. 